tionalisierung von Lesbenforschung und den damit verbundenen Gefahren einer Entradikalisierung und weiteren Entfernung von der Bewegung. Viel zu kurz kam die Diskussion darüber, was Lesbenforschung (und -bewegung!) denn nun eigentlich sei, wen/was sie umfaBt, welche Methoden sie anwendet, für wen sie forscht, was ihr Erkenntnisziel und -interesse sein soll. Grundlage dafür wäre allein schon die Diskussion darüber, wer/was alles unter den Begriff der Lesbe subsumiert werden soll. Auch das Thema der Differenz wurde letztlich nur angerissen, wenngleich sich dahinter der gröBte Konfliktstoff zu verbergen schien - wobei die aus der amerikanischen Diskussion übernommene Rede von der Ausklammerung "schwarzer und jüdischer« Frauen gelegentlich zur Formel zu verkommen drohte, hinter der sich das eigene elitäre Selbstverständnis, das auch die eigene Einschätzung als revolutionäre Avantgarde beinhaltet, verbarg.

Bleibt die - nicht zuletzt durch die von vielen konstatierte Bereitschaft einander zuzuhören - gestärkte Hoffnung auf eine produktive Fortsetzung der Auseinandersetzungen und eine Institutionalisierung wenigstens des Diskussionszusammenhanges. Eine zweite Tagung für 1992 wurde in jedem Fall schon einmal anvisier und konkret besprochen.
Brinja Sisenop, Annette Wolff

\section{Differenzen verdeutlichen - Grenzen respektieren?}

\author{
Bericht über die Tagung \\ "Integration und \\ Ausgrenzung weiblicher \\ Interessen in Politik und \\ Recht« vom 2.-7. Juli 1991 \\ in Bremen
}

Der Schwerpunkt der Tagung war von den Veranstalterinnen (Sabine Klein-Schonnefeld, Universität Bremen und Konstanze Plett, Zentrum für Europäische Rechtspolitik, Bremen) auf die rechtlichen, sozialen und kulturellen Unterschiede zwischen Frauen gelegt worden. Soziologinnen, Anthropologinnen und Juristinnen aus drei Kontinenten sprachen verschiedene Probleme aus ihren jeweiligen Arbeitsbereichen an.

Trotz der Beschränkung auf die Situation von Frauen aus den westlichen Industrienationen erwiesen sich die Diskussionen über Ausgrenzung und Integration als sehr komplex und heterogen.

Zunächst wurde die Frage nach sozialen und ökonomischen Lebensbedingungen von Frauen gestellt, die die implizite Grundlage des Profitierens von Gleichstellungs- und Gleichbehandlungspolitiken darstellen. Beispiele waren die Gleichstellungspolitik der EG, die Rechtsprechung des EuGH und die Stellung von Frauen im Erwerbsleben (Josephine Shaw, Universität Keele, Großbritannien). Dabei wurde deutlich, daß eine rechtlich-formale Gleichbehandlung selten die unterschiedlichen Ausgangspositionen und Lebenslagen von Frauen und Männern berücksichtigt. Im Gegenteil: führt diese Gleichstel- 
lungspolitik doch eher zum erhöhten Zwang, patriarchale Anforderungen und Strukturen zu akzeptieren und auszufüllen (z.B. Aufhebung des Nachtarbeitsverbotes).

Celia Davies, Inhaberin des ersten Frauenforschungslehrstuhls in Nordirland (University of Ulster), thematisierte anhand einer empirischen Studie, welche geschlechtsspezifischen Belastungen und Verzichte eine auf das Erwerbsleben beschränkte Integration Frauen auch dann abfordert, wenn diese Frauen in privilegierte Positionen vorstoßen. Das Aufzeigen von sozialen Differenzen zwischen Männem und Frauen sollte dabei nicht ins andere Extrem (der ausschlieBlichen Manifestation von Frau als Mutter) umschlagen. Davis stellte die Gleichheit von Frauen zur Diskussion und machte die Dynamik von Gleichheit, Assimilation und Unterdrückung deutlich. Gleichberechtigung führt danach zu einer Anpassung an einen männlichen Maßstab. Das Ideal ist hier immer noch der weiße, westliche, militarisierte, konkurrierende, individualistische Mann. Gleichbeit bedeutet also Integration in einen geschlechtlich aufgeteilten und definierten Raum (»gendered space $«$ ). Feministinnen müßten folglich nicht um Gleichheit kämpfen, sondern gegen die Unterdrückung unterschiedlicher Identitäten und der Vielfalt von Lebensqualitäten von Frauen. Unterscheidungen von Privatheit und Öffentlichkeit wurden als historisch und sozial konstruiert dargestellt. Die Macht liegt hier bei denen, die die Konstruktions- bzw. Definitionsgewalt haben, also den Männern, die sich die Aneignung von Frauen und Frauenarbeit im sogenannten privaten Sektor freigestellt haben.

Dieser Aspekt wurde auch von Minka de Soto, University of Madison, Wisconsin, aufgegriffen. Sie stellte in ihrem Beitrag die Beruicksichtigung von Frauen in der sozialistischen Theorie dar, wobei sie deutlich machte, daß die großen linken Denker klar zwischen Öffentlichkeit und Privatheit getrennt haben, und dabei die
Frau immer in die private Sphäre verwiesen wurde. Lediglich einige Sozialistinnen, wie z.B. Alexandra Kollontai, stellten diese Zuweisung in Frage. Entsprechend gestaltete sich die Situation von Frauen in der ehemaligen DDR, in der es eine staatliche Organisation für Frauen (DFD), jedoch nicht von Frauen gab. Dort wurde das Bild der Super-Frau gezeichnet, die Beruf/Karriere und Haushalt/Kinder mühelos vereinbaren kann. $\mathrm{Zu}$ Wende-Zeiten organisierten sich Frauen im Unabhängigen Frauenverband (UFV), die auch maBgeblich an den Diskussionen am Runden Tisch beteiligt waren, jedoch danach ins Abseits gestellt bzw. bei der ersten gesamtdeutschen Wahl völlig marginalisiert wurden. So blieben auch bier die angestrebten demokratischen Strukturen, in denen Frauen Ziele selbst bestimmen und entsprechende Strukturen formen, auf der Strecke. Daher bestand auch ein grober Bedarf, mit Frauen aus den neuen Bundesländern zu diskutieren. Die Interessienten mußten jedoch ihre Teilnahme absagen, da ihre Arbeitssituation gefährdet oder im Umbruch war.

Lisa Frohmann (American Bar Foundation, Chicago) legte anhand ihrer rechtssoziologischen Untersuchung dar, in welchem Umfang sozialer Status und ethnisch-kulturelle Zugehörigkeit den Zugang zum Rechtsschutz beeinflussen. Im USamerikanischen Strafrecht entscheidet bei Sexualdelikten zunächst eine Geschworenenjury daruiber, ob die Verfolgung - in diesem Falle des Vergewaltigungsdeliktes - in einem ordentlichen Gerichtsverfahren behandelt wird. Lisa Frohmann untersuchte speziell Delikte, bei denen Täter und Opfer eine enge soziale Beziehung zueinander hatten. Während der Diskussion wurde herausgearbeitet, wie herkömmlich patriarchale Heterosexualität formuliert wird, was als Konsens-Sexualität gilt und was als Zwangs-Sexualität. So wird z.B. nach der patriarchalen Rechtsauffassung bei jeder weiteren sexuellen Interaktion von einem Konsens ausgegangen, sozusagen von gegenseitigem Einverständnis, 
wenn die Frau zuvor eingewilligt hatte. Ebenso soll kein Zwang vorgelegen haben, wenn die Frau den Täter danach noch einmal freiwillig sieht oder die Beziehung fortsetzt. Insgesamt wurde festgestellt, daB vieles, was Frauen als Gewalt erfahren, im herrschenden Verständnis nicht als solche berücksichtigt wird. Die Definition von Konsens und Zwang innerhalb der heterosexuellen Beziehung richtet sich nach männlichen Normen und nicht nach Verletzbarkeit und Grenzen der Frauen. Die Notwendigkeit der Veränderung des Geschlechterverhältnisses innerhalb der Gesellschaft und der Annahmen über Sexualität seien somit notwendig, um die Mehrheit der Sexualdelikte nicht länger unberücksichtigt zu lassen.

Die Machtbeziehungen, die über die Definition von Sexualität hergestellt werden, müssen weiterhin ein wichtiger Schwerpunkt feministischer Diskussion bleiben. Kontakte, Standortbestimmungen und Selbst-Definitionen sind für Frauen dringend erforderlich. Die Bedeutung der differenzierten Analyse von geschlechtlichen Machtbeziehungen durch eine weitgestreute feministische Bewegung mit $\mathrm{Be}$ rücksichtigung der unterschiedlichen sozialen Kontexte wurden auch bei Nancy Matthews Sudie, Colgate University, zu rape-crisis-centers" (vergleichbar dem Notruf für vergewaltigte Frauen in der BRD) in den USA thematisiert. Hier stand die zusätzliche Problematik zur Debatte, auf welche Art und Weise und unter welchen inhaltlichen Zugeständnissen frauenspezifische Problemlagen in Normal-Institutionen eingegliedert werden können.

Damit wurde deutlich, wie einfach es ish, Differenzen zu ignorieren, und es wurde versucht, Ansätze der Veränderung zu finden. SchwerpunktmäBig ging es darum, die Mechanismen der Ungleichheit, Differenzen und Desintegration darzustellen. Als Beispiele dienten das deutsche Asylrecht und AusländerInnenrecht mit ihrer Ausgrenzung frauenspezifischer Fluchtgründe (Bettina Sokol, Richterin am Bremer Verwaltungsgericht). In diesem Kon- text wurde auch der implizite Assimilationsdruck thematisiert, der Konzepten der multikulturellen Gesellschaft innewohnt (Rose Baaba Folson, Universität Bremen).

Die Zerstörung des matrilinearen Systems der nordamerikanischen Indianerlnnen und die grundlegende Änderung der Stellung der Frau wurde von Bea Medicine (emeritierte Anthropologie-Professorin der Wakpala Universität, S.D., Kalifornien) dargestellt. Zugleich machte sie die Hoffnung deutlich, die durch zunehmende Beteiligung der indianischen Frauen am Rechtssystem $(1 / 3$ der indianischen RechtsanwältInnen sind Frauen), begründet wird, da diese versuchen, einige Elemente des matrilinearen Systems wieder zu stärken und damit Frauen größeren Einfluß zu verschaffen. Angestrebt wird nicht die Integration in das US-amerikanische Rechtssystem, sondern eine relative Autonomie des indianischen Systems, d.h. eine Abwehr der Vereinnahmung durch weiße diskriminierende Strukturen.

Ein weiterer Schwerpunkt war die Frage nach den politischen, gesetzlichen und theoretischen Konsequenzen einer so verstandenen Differenzdebatte, die sich primär auf die unterschiedlichen Lebenslagen und -entwürfe von Frauen selbst bezieht. Hier wurde auch der Mythos der Gleichheit aller Frauen in Frage gestellt und heftig diskutiert.

Dabei wurde die Notwendigkeit festgestellt, Kultur als Widerstandsform von Frauen zu begreifen. So benutzen z.B. in Südamerika Frauen Religion als eine Möglichkeit des Aufbegehrens und drükken so ihre Macht aus, die bisher unsichtbar geblieben war. Daß herrschende Kultur dagegen patriarchale Unterdrückung und Ausgrenzung von Frauen repräsentiert, verdeutlichte Mari Ann Rossi (Soziologin an der Universität Wisconsin) anhand ihrer Forschung über die Stellung der Frau im Katholizismus: Gelte doch immer noch die Projektion der duldsamen, gefügig ergebenen (Ehe)Frau, die eins mit dem Mann - sich völlig aufgebe. Falls sie dies nicht tue, werde sie dazu 
gezwungen, wobei Schläge, Vergewaltigungen etc. immer noch legitime Mittel darstellten.

Wichtig war es, die Differenzen zwischen Frauen aufzuzeigen, jedoch den Dialog nicht auszuschlieBen, und durch Darstellung subjektiver Standpunkte einen Austausch zu ermöglichen und Verständnis zu schaffen. Dies war und ist ein schwieriger ProzeB. Er setzt nicht nur die
Motivation voraus, Differenzen unter Frauen anzuerkennen und zu erforschen, sondern erfordert ein Offenlegen persönlicher Betroffenheiten sowie die Bereitschaft, subjektive Erfahrungen, Wertungen und Ziele in die wissenschaftliche Auseinandersetzung einzubeziehen. Dieser ProzeB stell die Dichotomie zwischen sogenanntem Privaten und Öffentlichen/Wissenschaftlichen in Frage. 